\title{
Article \\ Optical Orientation and Inverse Spin Hall Effect as Effective Tools to Investigate Spin-Dependent Diffusion
}

\author{
Marco Finazzi ${ }^{1, *}$, Federico Bottegoni ${ }^{1}$, Carlo Zucchetti ${ }^{1}$, Monica Bollani ${ }^{2}$, Andrea Ballabio ${ }^{1}$, \\ Jacopo Frigerio ${ }^{1}$, Fabien Rortais ${ }^{3,4}$, Céline Vergnaud ${ }^{3,4}$, Alain Marty ${ }^{3,4}$, Matthieu Jamet ${ }^{3,4}$, \\ Giovanni Isella ${ }^{1}$ and Franco Ciccacci ${ }^{1}$ \\ 1 Dipartimento di Fisica and LNESS, Politecnico di Milano, Piazza Leonardo da Vinci 32, 20133 Milano, \\ Italy; federico.bottegoni@polimi.it (F.B.); carlo.zucchetti@polimi.it (C.Z.); andrea.ballabio@polimi.it (A.B.); \\ jacopo.frigerio@polimi.it (J.F.); giovanni.isella@polimi.it (G.I.); franco.ciccacci@polimi.it (F.C.) \\ 2 Istituto di Fotonica e Nanotecnologie - Centro Nazionale delle Ricerche, LNESS, via Anzani 42, \\ 22100 Como, Italy; monica.bollani@ifn.cnr.it \\ 3 Université Grenoble Alpes, Institut Nanosciences et Cryogénie (INAC) - Spintec, F-38000 Grenoble, \\ France; fabienrortais@gmail.com (F.R.); celine.vergnaud@cea.fr (C.V.); alain.marty@cea.fr (A.M.); \\ matthieu.jamet@cea.fr (M.J.) \\ 4 Commissariat à l'énergie atomique et aux énergies alternatives (CEA), Institut Nanosciences et Cryogénie \\ (INAC) - Spintec, F-38054 Grenoble, France \\ * Correspondence: marco.finazzi@polimi.it; Tel.: +39-02-2399-6177
}

Academic Editors: Matteo Cantoni, Riccardo Bertacco and Christian Rinaldi

Received: 1 October 2016; Accepted: 17 November 2016; Published: 22 November 2016

\begin{abstract}
In this work we address optical orientation, a process consisting in the excitation of spin polarized electrons across the gap of a semiconductor. We show that the combination of optical orientation with spin-dependent scattering leading to the inverse spin-Hall effect, i.e., to the conversion of a spin current into an electrical signal, represents a powerful tool to generate and detect spin currents in solids. We consider a few examples where these two phenomena together allow addressing the spin-dependent transport properties across homogeneous samples or metal/semiconductor Schottky junctions.
\end{abstract}

Keywords: optical orientation; spin current; inverse spin Hall effect

\section{Introduction}

Spintronics (spin transport electronics or spin-based electronics) consists in the study and, possibly, in the active control of spin degrees of freedom in solid-state systems. Among the goals of spintronics is to understand how the particle spin interacts with its solid-state environments determining phenomena such as spin transport, dynamics, and relaxation [1]. In this frame, the production of a spin current, i.e., a net flow of spins, in nonmagnetic materials and, in particular, semiconductors is a topic of ever-growing importance because of the implications it might have for applications concerning the manipulation of spin in electronic devices. Noteworthy, spin transport can be completely decoupled from charge transport, and it is in principle possible to generate currents of spins only, with no net particle transfer along the channel. Such a pure spin current is invariant under time reversal: if the clock is ran backward, the spin current would still flow in the same direction. This feature ensures that quantum information can, in principle, be sent through the channel without losing quantum coherence. Moreover, a device relying on pure spin transport should have faster switching times and lower power consumption than conventional devices, mainly because spins can be manipulated faster and at lower energy cost than charges can. 
Techniques able to generate a (pure) spin current typically induce a space-dependent spin-split electrochemical potential across the sample resulting in the generation of carriers with a prevailing spin orientation. The most common ones are (i) precession-induced spin pumping [2,3]; (ii) spin injection from ferromagnetic materials [4-6]; and (iii) optical orientation in semiconductors $[7,8]$. The latter exploits the spin-orbit interaction, which allows inducing a net spin polarization by manipulating the electron orbital degrees of freedom with circularly polarized light. Spin-orbit removes the energy degeneracy between heavy and light holes (HH and $\mathrm{LH})$ states and split off (SO) states of the valence band at the $\Gamma$ point of the semiconductor Brillouin zone: a net electron spin polarization is obtained when the photon energy is tuned to the direct bandgap $E_{d}$ and electrons coming only from $\mathrm{HH}$ and LH states are promoted to the conduction band. Although the first demonstration of optical orientation was done in Si [7], the relatively small splitting between $\mathrm{HH}-\mathrm{LH}$ and $\mathrm{SO}$ states $\left(\Delta_{\mathrm{so}}=44 \mathrm{meV}\right.$ in $\mathrm{Si}$ ) represents a demanding constraint to avoid excitation of electrons from SO states. This limitation together with the indirect nature of the Si electronic gap implies that in such a material only a very small spin polarization $P=\left(n_{\uparrow}-n_{\downarrow}\right) /\left(n_{\uparrow}+n_{\downarrow}\right)$ is achievable, where $n_{\uparrow}\left(n_{\downarrow}\right)$ are the up (down) spin densities (as referred to the quantization axis parallel to the light wavevector). On the other hand, the SO splitting is much larger in semiconductors such as $\mathrm{Ge}$ and GaAs, which are characterized by a higher atomic number $\mathrm{Z}$ than $\mathrm{Si}$ $\left(\Delta_{\mathrm{Ge}} \approx 0.29 \mathrm{eV}\right.$ and $\Delta_{\mathrm{GaAS}} \approx 0.34 \mathrm{eV}$, respectively), allowing the excitation of an electron population in the conduction band of these semiconductors with a spin polarization $P$ that can be as high as $50 \%$ for bulk materials $[9,10]$, but that can reach even higher values in low symmetry systems where the HH-LH degeneracy is removed by e.g., strain [11-13] or reduced dimensionality [14]. In addition to allowing the generation of much higher spin polarizations in semiconductors as compared with all-electrical spin-injection schemes, spin orientation can also be exploited to create a spatially-modulated spin voltage beneath a patterned overlayer and to realize the spin analogue of a photovoltaic generator [15].

In the following, we will show that optical orientation represent an effective tool to generate sizable spin currents and spin-split electrochemical potentials in semiconductors. We will also demonstrate how such optically-induced spin currents can be exploited to understand spin transport and relaxation both in the semiconductors where they are generated and in other nonmagnetic materials in which the spin current can be injected. In particular, the focus will be on optical orientation in either $\mathrm{GaAs}$ or $\mathrm{Ge}$. In these two materials the electron spin lifetime is in the nanosecond range [16,17], whereas the hole spin lifetime is much shorter (few hundreds of femtoseconds [18,19]), therefore spin transport phenomena in GaAs and Ge can be assumed to be associated mainly with electrons. While GaAs is characterized by a conduction band minimum at $\Gamma$, the spin-oriented photoelectrons excited at the bottom of the Ge conduction band at $\Gamma$ are scattered to the $\mathrm{L}$ minima within about $300 \mathrm{fs}$, partially maintaining the initial spin orientation [17]. Another feature distinguishing spin transport in these materials is the lack of inversion symmetry of GaAs, while Ge is centro-symmetric. This means that $\mathrm{Ge}$ is immune from the spin-relaxation channel associated with the Dyakonov-Perel mechanism [20], which is instead active in GaAs [21,22].

Since spin is not a conserved quantity, detecting spin currents has proven a very elusive task. One solution is to perform a spin-resolved photoemission experiment where the electrons excited in the conduction band are extracted into the vacuum after the realization of negative or near-negative electron affinity conditions at the surface of the semiconductor $[7,9,11,13,23]$. This technique has the advantage of providing absolute values of the spin polarization of the photo-emitted electrons, which, however, might be affected by spin depolarization occurring when they are transferred from the bulk of the sample to the surface and then to vacuum. Another solution is represented by optical techniques exploiting the transfer of angular momentum between the photons and the spin-polarized electrons, leading to Faraday or Kerr rotation of the linear polarization of the light. Unfortunately such methods are characterized by very low signals, require low temperatures, and are diffraction limited. One might also measure the circular polarization of the photoluminescence light 
emitted when spin-polarized conduction electrons radiatively recombine across the gap [17,24,25]. Spin accumulation might be locally probed by measuring the current crossing magnetic tunnel junctions (MTJs) fabricated by lithography techniques where a ferromagnetic layer is separated from the semiconductor by a thin insulating spacer [26]. MTJ have the advantage that they can be fabricated to have either in-plane or out-of-plane magnetic anisotropy and be sensitive to any component of the spin polarization.

In the following we will adopt an alternative method with respect to those described above and exploit the inverse spin Hall effect (ISHE) illustrated in Figure 1. This phenomenon consists in the spin-dependent scattering induced by the spin orbit interaction and results in the conversion of a spin current into an electric signal by generating electromotive field $E_{\text {ISHE }}$ in the material in which spin-polarized charges are flowing [27]. $E_{\text {ISHE }}$ is given by the following expression [28]:

$$
\mathbf{E}_{\mathrm{ISHE}}=\frac{\gamma}{\sigma_{c}} \mathbf{J}_{s} \times \mathbf{P},
$$

where $\gamma$ and $\sigma_{c}$ represent the spin-Hall angle and the electrical conductivity of the material, respectively, and $\mathbf{P}$ is the spin polarization vector defined as $\mathbf{P}=\left(n_{\uparrow}-n_{\downarrow}\right) /\left(n_{\uparrow}+n_{\downarrow}\right) \mathbf{u}_{k}, \mathbf{u}_{k}$ being the unit vector parallel to the quantization axis (which is taken parallel to the light propagation axis when the spins are generated by optical orientation).

Such a spin-to-charge current conversion can occur either in the same semiconductor where optical orientation is achieved $[29,30]$ or inside a high- $Z$ metal layer, such as $\mathrm{Pt}, \mathrm{Au}$ or $\mathrm{Bi}$, deposited on the semiconductor surface [31,32], in which the spin-polarized electrons produced by optical orientation are injected. In this second case, one can exploit the sizable value of the spin Hall angle $\gamma$ of the high- $Z$ material, which can be thus exploited as a non-magnetic electrode sensitive to pure spin-currents able to provide larger $E_{\mathrm{ISHE}}$ signals than those that could be measured directly in the semiconductor.

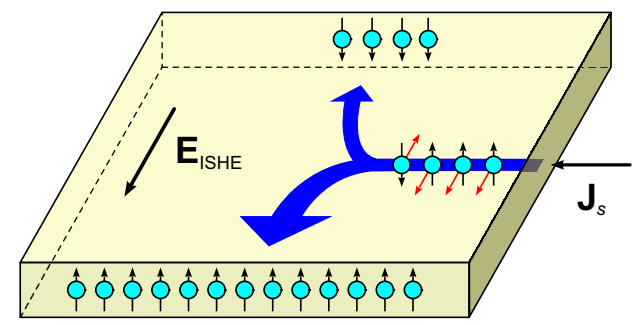

Figure 1. Inverse spin-Hall effect. A spin polarized current density $\mathbf{J}_{s}$ undergoes asymmetric spin-orbit-induced scattering. The charges accumulated on the sides of the conductor generate an electromotive field $\mathbf{E}_{\mathrm{ISHE}}$.

\section{Experimental}

The experimental geometry for optical orientation/ISHE detection is illustrated in Figure 2. The polarization state of a collimated laser beam illuminating the semiconductor surface is modulated between right- and left-circular polarization by a photoelastic modulator at $50 \mathrm{kHz}$. The photoinduced electromotive force $\Delta V_{\mathrm{ISHE}}$ associated with $\mathrm{E}_{\mathrm{ISHE}}$ is measured between two $100 \mathrm{~nm}$-thick $\mathrm{Au} / \mathrm{Ti}$ contacts deposited at the edges of the sample surface and is demodulated by a lock-in amplifier in open circuit conditions. This modulation/demodulation scheme allows rejecting any possible spurious photovoltaic signal, which should depend only on the light intensity and be insensitive to the light helicity. When a nonmagnetic film is used as an ISHE electrode, as in Figure 2, a $4 \mathrm{~nm}$-thick Pt layer is grown by e-beam evaporation prior to the fabrication of the $\mathrm{Au} / \mathrm{Ti}$ electrodes.

From Equation (1), one readily obtains that a net photoinduced spin polarization can be detected only when, referring to Figure 2, $\mathbf{P}$ has a nonvanishing component along the $x$ axis: in this case, 
$\Delta V_{\text {ISHE }}$ depends only on the spin polarization along $x$. More precisely, the dependence of $\Delta V_{\text {ISHE }}$ on the set-up geometry is given by the following expression:

$$
\Delta V_{\text {ISHE }} \propto t_{s} t_{p} \cos \delta \cos \phi \tan \theta P_{\text {circ }}
$$

with $P_{\text {circ }}$ being the degree of circular polarization of the light impinging on the sample surface, $t_{s(p)}$ the transmission coefficient of the $s(p)$-polarized light, and $\delta$ the angle between the direction of the light inside the semiconductor and the normal to the sample surface [33]. A multiaxial stage allows the rotation around the polar angle $\theta$ and the azimuthal angle $\phi$ as defined in Figure 2. In the following, all ISHE data have been collected at $\theta=65^{\circ}$ and $\phi=0$.

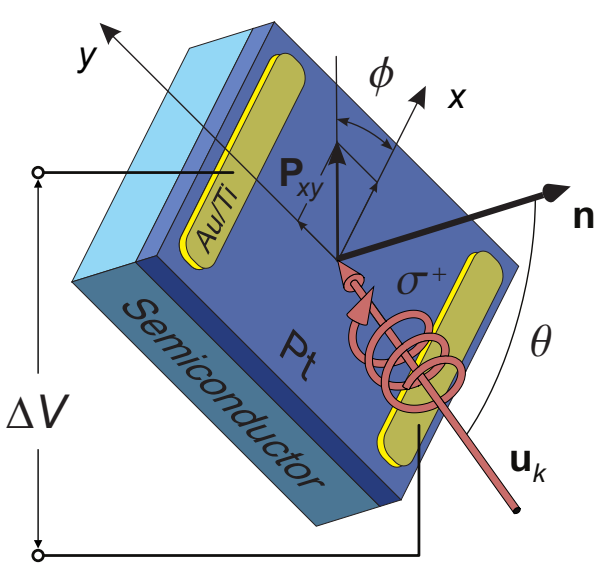

Figure 2. Scheme of the optical orientation/ISHE geometry in the case a Pt layer is deposited on top of the semiconductor (Ge or GaAs): $\theta$ is the angle between the direction of the incident light $\mathbf{u}_{k}$ and the normal $\mathbf{n}$ to the sample, $\phi$ is the angle between the projection of $\mathbf{u}_{k}$ in the sample plane and the $x$ axis. The demodulated signal $\Delta V$ is sensitive to the component of the spin polarization along the in-plane $x$ axis.

\section{Results and Discussion}

\subsection{Extrinsic spin-Hall Conductivity in Si-doped GaAs}

The first example that we present consists in the experimental determination of the spin-Hall angle $\gamma$ in a heavily Si-doped GaAs(001) sample (donor concentration $N_{\mathrm{Si}}=2 \times 10^{18} \mathrm{~cm}^{-3}$ ). As previously outlined, the charge/spin conversion mechanism described in Figure 1 has been experimentally shown to be active in $n$-doped III-V semiconductors, where spin-dependent electron scattering can lead to a transverse spin accumulation (known as spin Hall effect or SHE) at the sample edges when a charge current is flowing [34-36], or, conversely, to ISHE when a spin current is injected into the system $[29,37]$. Since these phenomena are attributed to scattering with impurities and not to intrinsic properties of the material, they are referred to as extrinsic spin-Hall effects.

Figure 3a shows the $\Delta V_{\mathrm{ISHE}}$ signal measured on a Si-doped $\left(N_{\mathrm{Si}}=2 \times 10^{18} \mathrm{~cm}^{-3}\right) \mathrm{GaAs}$ sample [30]. The data were collected by illuminating the sample with the collimated light (spot diameter $d \approx 3 \mathrm{~mm}$ ) from a Ti:Sapphire tunable laser in the 1.4-1.85 eV photon energy range. By solving the drift-diffusion equation for spin, one obtains the following expression [30]:

$$
\Delta I_{\mathrm{ISHE}}=\frac{\Delta V_{\mathrm{ISHE}}}{R}=\gamma \frac{G_{0} L_{s}^{2}}{1+\alpha L_{s}} d,
$$

where $R=660 \Omega$ is the resistance measured between the $\mathrm{Au} / \mathrm{Ti}$ contacts, $\alpha$ is the GaAs absorption coefficient, and $G_{0}$ is the number of spin-polarized electrons generated per unit time and unit volume in GaAs. In principle, this expression allows determining the value of the spin-Hall angle $\gamma$ from the experimental ISHE data of Figure 3a. This is possible if one could estimate the spin diffusion length 
$L_{s}=\sqrt{D_{e} \tau_{s}}$, with $D_{e}=0.022 \mathrm{~m}^{2} \mathrm{~s}^{-1}$ being the electron diffusion coefficient and $\tau_{s}$ the spin relaxation time, respectively. The value of the latter can be estimated by considering the two main relaxation mechanisms operating at $T=300 \mathrm{~K}$, namely polar optical phonon and ionized impurity scattering, as described in Ref. [30].

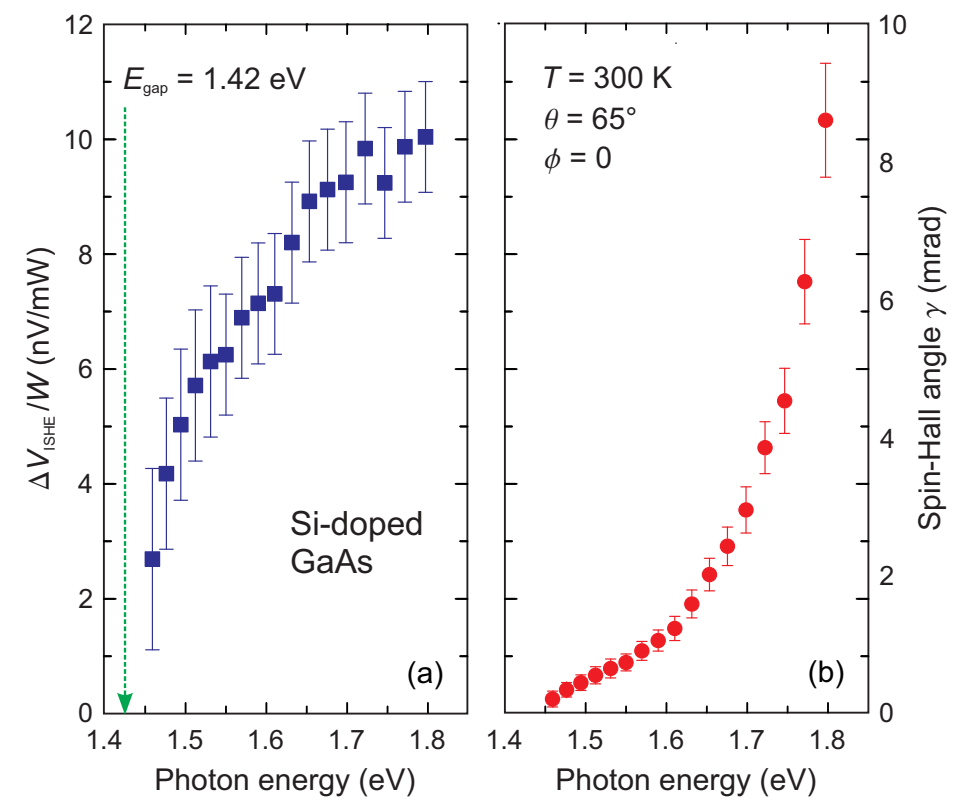

Figure 3. (a) Room temperature ISHE signal in Si-doped GaAs as a function of the incident photon energy in the 1.42-1.8 eV range for $\theta=65^{\circ}$ and $\phi=0$. The values are normalized with respect to the power $W$ entering into the GaAs sample (evaluated with a GaAs reflectivity equal to 0.34 ); (b) Room temperature spin-Hall angle $\gamma$, extrapolated from panel (a).

Spin-dependent electron scattering from impurities can be described in terms of Mott skew scattering [38,39], as already suggested by Engel et al. for Si-doped GaAs [40], and by side jumps [41], a phenomenon consisting in spin-dependent lateral displacements of the single-particle wave packet at each scattering event. When these mechanisms are accounted for in a system kept at room temperature [30], one obtains the spin-Hall angle as a function of the photon energy reported in Figure $3 \mathrm{~b}$. Values for $\gamma$ in heavily $n$-doped GaAs at room temperature have not been previously reported in the literature, however the value of $\gamma=(2 \pm 1) \times 10^{-4}$ that we estimate at room temperature for photon energies almost resonant with the GaAs energy band gap is roughly one order of magnitude lower than the one theoretically predicted [40] and experimentally measured $[34,42]$ at low temperatures.

This results highlight the role of the different spin-relaxation mechanism at high temperature and, at the same time, demonstrate the extreme sensitivity of the spin current detection scheme based on the ISHE effect with respect to scanning Kerr microscopy measurements such as those reported in Refs. [34,42], which are not able to measure significant signals associated with spin accumulation at room temperature.

\subsection{Photon Energy Dependence of the Optically-Induced Inverse spin-Hall Effect in Pt/GaAs and Pt/Ge}

As mentioned in Section 1, an alternative route to investigate spin currents generated in semiconductors consists in measuring the ISHE signal across a film of a high- $Z$ material such as $\mathrm{Pt}$ deposited on top of the semiconductor. In addition to providing a means to detect spin currents with better sensitivity thanks to the more efficient spin-dependent scattering in such materials associated with a large spin-orbit coupling, these systems allow studying spin transport across a metal/semiconductor interface, which represent an extremely important benchmark in spintronics. 
An example is reported in Figure 4, showing optically generated ISHE in $\mathrm{Pt} / \mathrm{GaAs}$ and $\mathrm{Pt} / \mathrm{Ge}$ (dots). The samples consist in a $350 \mu \mathrm{m}$-thick Si-doped GaAs wafer (donor concentration $\left.N_{\mathrm{Si}} \approx 2 \times 10^{18} \mathrm{~cm}^{-3}\right)$ and a $450 \mu \mathrm{m}$-thick As-doped Ge wafer $\left(N_{\mathrm{As}} \approx 1.7 \times 10^{16} \mathrm{~cm}^{-3}\right)$. Both are covered with a $4 \mathrm{~nm}$-thick $\mathrm{Pt}$ film on which the $\mathrm{Au} / \mathrm{Ti}$ electrodes are directly grown [43]. Since the aim of the following discussion is a comparative study of the trends describing the photon energy dependence of the ISHE signal in the two semiconductors, the data in Figure 4 are normalized to the respective maximum $\Delta V_{\text {ISHE }}$ values.
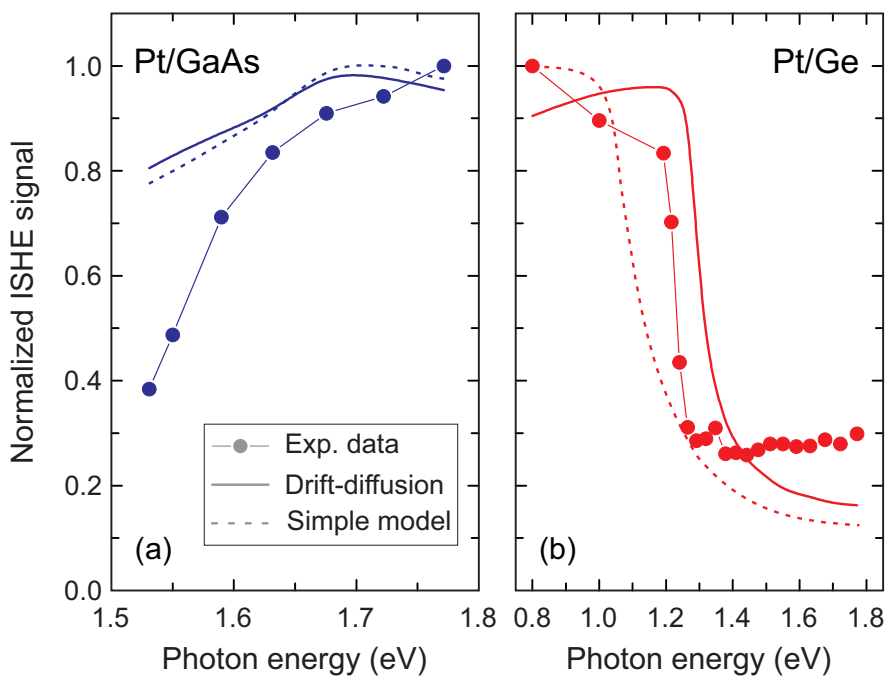

Figure 4. Comparison between the measured ISHE signal (obtained at $\theta=65^{\circ}$ and $\phi=0$ ) and the calculated spin current. The full lines correspond to the result of the drift-diffusion model, while the dashed lines to the simplified model assuming that all the spins generated within a distance $L_{S}$ from the metal/semiconductor interface are injected in the Pt contact (see text). (a) Pt/GaAs $\left(\tau_{s}=10^{-11} \mathrm{~s}\right)$; (b) $\mathrm{Pt} / \mathrm{Ge}\left(\tau_{\mathrm{s}}=10^{-9} \mathrm{~s}\right)$.

$\mathrm{Pt} / \mathrm{GaAs}$ and $\mathrm{Pt} / \mathrm{Ge}$ show qualitatively different photon energy dependences of the ISHE signal. While $\Delta V_{\text {ISHE }}$ increases with $h v$ for Pt/GaAs in the energy range between the GaAs gap $\left(E_{\mathrm{GaAs}}=1.43 \mathrm{eV}\right)$ and the threshold for split-off state excitation $\left(E_{\mathrm{GaAs}}+\Delta_{\mathrm{GaAs}}=1.77 \mathrm{eV}\right)$, in agreement with the modeling simulation of Ref. [44], in Pt/Ge the ISHE signal is maximum at the Ge direct gap $\left(E_{\mathrm{Ge}}=0.8 \mathrm{eV}\right)$ and abruptly decreases when the photon energy is increased above $E_{\mathrm{Ge}}+\Delta_{\mathrm{Ge}}=1.1 \mathrm{eV}$.

To better understand these trends, we have numerically solved the $1 \mathrm{D}$ drift-diffusion Poisson equations for spin and charge currents in the semiconductors [43]. The electron mobility at the doping density of our samples was assumed equal to $\mu_{\mathrm{Ge}}=3560 \mathrm{~cm}{ }^{2} \cdot \mathrm{V}^{-1} \cdot \mathrm{s}^{-1}$ and $\mu_{\mathrm{GaAs}}=2051 \mathrm{~cm}^{2} \cdot \mathrm{V}^{-1} \cdot \mathrm{s}^{-1}$ [45], corresponding to a diffusion coefficient $D_{e}=\mu k_{\mathrm{B}} T / e$ approximately equal to $89 \mathrm{~cm}^{2} \cdot \mathrm{s}^{-1}$ for Ge and $50 \mathrm{~cm}^{2} \cdot \mathrm{s}^{-1}$ for GaAs, respectively. The generation term was explicitly accounted for by considering the semiconductor absorption coefficient [46] and the spin polarization theoretically expected for illumination with $100 \%$ circularly polarized light $[47,48]$, while carrier recombination was described by the Shockley-Read term [49] and spin relaxation by a decay time $\tau_{s}$. The model portrays the spatial distribution of spins residing at the $\Gamma$ valley for GaAs and at the $L$ valleys for $\mathrm{Ge}$ under the approximation that all the electrons are thermalized. This also implies that the spin relaxation time $\tau_{s}$ does not depend on the photon energy. Boundary conditions where chosen to have $J_{s}=0$ in the semiconductor bulk and a vanishing density of spin-polarized carriers at the $\mathrm{Pt}$ interface. This last assumption is justified by the spin diffusion length $L_{S}$ being much smaller in $\mathrm{Pt}$ than in Ge or GaAs. The calculation is carried on as a function of $\tau_{s}$. The results are shown in Figure 4 by the full lines, obtained for $\tau_{s}=10^{-11} \mathrm{~s}$ (corresponding to $L_{s}=\sqrt{D_{e} \tau_{s}} \approx 0.22 \mu \mathrm{m}$ ) and $\tau_{s}=10^{-9} \mathrm{~s}$ $\left(L_{s} \approx 3 \mu \mathrm{m}\right)$ for GaAs and $\mathrm{Ge}$, respectively. From Figure 4 one can see that the model reproduces the 
qualitative trends of the ISHE signal and that the fitting correctly obtains a spin relaxation time for Ge larger than the one for GaAs, even if it underestimates the latter with respect to the value reported in the literature [16].

To gain further insight into the physical mechanisms responsible of the trends shown in Figure 4, we have also evaluated a much simpler model assuming that all the spins generated within a distance $L_{s}$ from the metal/semiconductor interface are injected in the Pt contact. In this case the spin current density $J_{s}$ can be written as follows [43]:

$$
J_{s}=q \Phi_{0} P(\hbar v)\left[1-e^{-\alpha(\hbar v) L_{s}}\right]
$$

with $\Phi_{0}$ being the photon flux into the semiconductor, $P(\hbar v)$ the initial polarization of the photo-generated electrons [47,48] and $\alpha(\hbar v)$ the absorption coefficient [46].

Figure 4 also shows the comparison between the experimental data and the ISHE signal obtained by using this simplified model (dashed lines). As apparent from the figure, the simplified model is still in good qualitative agreement with the experimental data and is even better than the drif-diffusion model in reproducing the decreasing trend observed for Ge at photon energies below $E_{\mathrm{Ge}}+\Delta_{\mathrm{Ge}}$. Since in Equation (4) all electric effects are completely neglected, our findings might indicate that the effects of the electric field within the depletion region are overestimated in the drift-diffusion model and a more refined analysis including image force lowering of the barrier and the presence of surface states should be considered [43].

\subsection{Pt Thickness Dependence of the Optically-Induced Inverse spin-Hall Effect in Pt/Ge}

As a last example we discuss the dependence of the ISHE signal measured in a Pt layer when an optically generated spin current is injected from a Ge substrate, as a function of the Pt thickness. This allows addressing different spin diffusion regimes in $\mathrm{Pt}$, highlighting the role of the $\mathrm{Pt} /$ semiconductor interface [50].

A $450 \mu \mathrm{m}$-thick As-doped Ge(001) substrate $\left(N_{\mathrm{As}} \approx 1.7 \times 10^{16} \mathrm{~cm}^{-3}\right)$ was first cleaned in acetone and isopropyl alcohol in an ultrasonic bath for $5 \mathrm{~min}$. It was then rinsed in deionized water before being loaded into the vacuum chamber (base pressure: $10^{-11} \mathrm{mbar}$ ). The native Ge oxide top layer was then thermally removed by annealing at $450{ }^{\circ} \mathrm{C}$ for $30 \mathrm{~min}$ and at $850{ }^{\circ} \mathrm{C}$ for $3 \mathrm{~h}$. At the end of the cleaning procedure, the reflection high-energy electron diffraction pattern from the $\mathrm{Ga}(001)$ surface showed a well-defined and high quality $(2 \times 1)$ surface reconstruction. At this point a polycrystalline $\mathrm{Pt}$ wedge (thickness ranging between 1 and $20 \mathrm{~nm}$ ) was grown by e-beam evaporation [50]. The ISHE signal was then collected by illuminating the sample with a coolimated laser beam at $h v=1.77 \mathrm{eV}$, keeping the sample at room temperature.

The ISHE signal normalized to the power $W$ of the light entering the Ge substrate is plotted as a function of the Pt thickness $t_{\mathrm{Pt}}$ in Figure $5 \mathrm{a}$ and shows an abrupt decrease above $10 \mathrm{~nm}$, partially due to a reduction of the resistivity of the $\mathrm{Pt}$ film. However, this rapid reduction is not canceled when the film conductivity $\sigma_{c}$ is explicitly accounted for to derive the $W$-normalized value of $J_{\mathrm{ISHE}}=\sigma_{c} \Delta V_{\mathrm{ISHE}} /\left(d \cdot W_{\mathrm{Ge}}\right)$, displayed in Figure $5 \mathrm{~b}[50]$.

We tried to describe the Pt thickness dependence of JISHE within a unidimensional model for spin diffusion along the axis perpendicular to the sample surface [31]. The fitting function resulting from this model is the following:

$$
J_{\mathrm{fit}}=\gamma J_{\mathrm{so}} \frac{L_{s}}{t_{\mathrm{Pt}}} \tanh \left(\frac{t_{\mathrm{Pt}}}{2 L_{s}}\right)
$$

which is a function of the free fitting parameters $\gamma J_{s 0}$ and $L_{s}$. From Figure $5 \mathrm{~b}$ one can see that a model considering a constant spin diffusion length $L_{s}$ is not able to fit the data over the whole $\mathrm{Pt}$ thickness range. The data points with $t_{\mathrm{Pt}}<10 \mathrm{~nm}$ can be fitted with $L_{s}=3.5 \pm 2.1 \mathrm{~nm}$, while those with $t_{\mathrm{Pt}}>10 \mathrm{~nm}$ all lie along the fitting curve obtained for $L_{s}=8.2 \pm 3.5 \mathrm{~nm}$. By assuming 
that $J_{s 0}$, corresponding to the spin current density at the Pt/Ge interface, remains constant over the entire $\mathrm{Pt}$ thickness range, we find that the spin-Hall angle $\gamma$ increases by more than a factor three for $t_{\mathrm{Pt}}<10 \mathrm{~nm}$. This noteworthy result suggests that either spin transport cannot be interpreted in terms of diffusion for a thickness comparable with the spin diffusion length, or that interface scattering plays an important role in determining the spin-Hall angle $\gamma$ [50]. Indeed, spin-dependent scattering is expected to be more efficient at the interface with a high- $Z$ material because of the broken inversion symmetry and the roughness.

Finally, we report in Figure $5 \mathrm{c}$ the $t_{\mathrm{Pt}}$ dependence of the $W$-normalized $I_{\text {ISHE }}=\Delta V_{\text {ISHE }} / R$ values, $R$ being the resistance measured across the $\mathrm{Au} / \mathrm{Ti}$ contacts. The non monotonic trend shown in Figure $5 \mathrm{c}$ is a further confirmation that a model based on constant values of the spin diffusion length $L_{s}$ and of the spin-Hall angle $\gamma$ fails in describing spin transport across the Pt layer, since Equation (5) would suggest a monotonic behavior, with $I_{\mathrm{ISHE}}$ increasing with $t_{\mathrm{Pt}}$. We would like to remind that a similar result has already been obtained in a hybrid yttrium iron garnet/platinum system [51], displaying a $I_{\text {ISHE }}$ signal peaking at $t_{\mathrm{Pt}} \approx 10 \mathrm{~nm}$. All these results point towards a drastic change in the spin transport properties in platinum thin films as function of thickness.
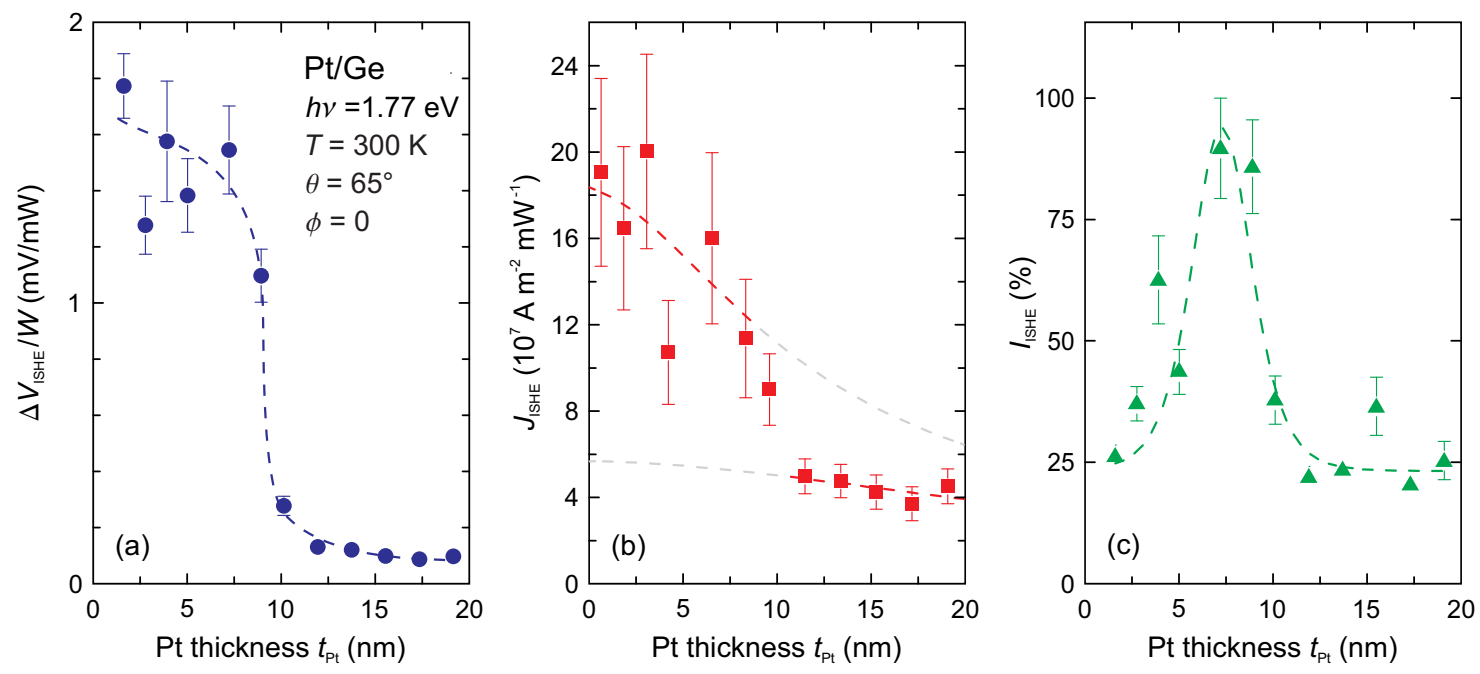

Figure 5. (a) ISHE signal measured at $h v=1.77 \mathrm{eV}$ in a $\mathrm{Pt} / \mathrm{Ge}$ sample kept at room temperature as a function of the Pt thickness $t_{\mathrm{Pt}}$ (dashed line: guide for the eye). Geometrical parameters: $\theta=65^{\circ}$ and $\phi=0$. The values are normalized to the power $W$ entering into the Ge substrate; (b) Inverse spin-Hall current density $J_{\mathrm{ISHE}}=\sigma_{c} \Delta V_{\mathrm{ISHE}} /\left(d \cdot W_{\mathrm{Ge}}\right)$ (squares), normalized to the transmitted power $W$ as a function of the Pt thickness. The lower dashed line represents the fitting function for $t_{\mathrm{Pt}}>10 \mathrm{~nm}$ calculated using Equation (5) with the free parameters $L_{s}=8.2 \pm 3.5 \mathrm{~nm}$ and $\gamma J_{s 0}=(1.1 \pm 0.2) \times$ $10^{8} \mathrm{~A} \cdot \mathrm{m}^{-2} \cdot \mathrm{mW}^{-1}$. The upper dashed line corresponds to the fitting function of Equation (5) for $t_{\mathrm{Pt}}<10 \mathrm{~nm}$ with the free parameters $L_{s}=3.5 \pm 2.1 \mathrm{~nm}$ and $J_{s 0}=(3.7 \pm 0.6) \times 10^{8} \mathrm{~A} \cdot \mathrm{m}^{-2} \cdot \mathrm{mW}^{-1}$; (c) Normalized $I_{\text {ISHE }}=\Delta V_{\text {ISHE }} / R$ dependence as a function of the Pt thickness: the maximum value is obtained for $t_{\mathrm{Pt}} \approx 10 \mathrm{~nm}$, in agreement with the ISHE measurements of Ref. [51].

\section{Discussion}

The cases discussed in the previous sections, and the physical insight in spin transport phenomena they provide, confirm that combining the generation of a spin current in a semiconductor through optical orientation together with spin detection by ISHE represents a viable pathway to investigate spin diffusion in solids. However, the joint exploitation of these two techniques is a rather young research field and there are many open issues that still deserve further investigations to reach a full understanding. In particular, the transfer of optically-oriented electrons across a metal/semiconductor junction presents many aspects that still lack an explanation. 
One example is represented by the fact that, in experiments similar to those reported Section 3.2, we were able to measure a sizable ISHE signal only when the semiconductor is $n$-doped, with signals below the detection limit in the opposite case of $p$-doping. This would suggest that the presence of a Schottky barrier is an important condition to realize an efficient transfer of optically-oriented electrons. This requirement reminds of the conductivity mismatch condition that needs to be fulfilled in spintronics to obtain an efficient spin injection from a ferromagnetic material into a semiconductor [6,52]. In this case, the spin polarization of the injected current can be significant only when the interface resistance exceeds a threshold value related to the resistivity and spin diffusion length of the semiconductor [6]. However, this conductivity mismatch problem arises only when one forces a spin current from a high-conductance (the ferromagnetic metal) to a low-conductance (the semiconductor) material. The same diffusion model applied in Ref. [6] to explain this effect is not able to justify the necessity of the presence of the Schottky barrier to ensure an efficient spin transfer from the high resistivity semiconductor, where spin-polarized electrons are created by optical orientation, to the low resistivity high- $Z$ metal.

To better address the influence of the Schottky barrier on the spin diffusion across the interface between a metal and a semiconductor, one might also study how $\mathbf{J}_{s}$ depends on the bias voltage. With respect to changing the doping concentration, this approach would have the significant advantage of allowing one to continuously tune the height of the Schottky barrier of the same interface, thus limiting the dispersion of the results that might be associated with different samples, surface preparation, and growth conditions. Investigation is currently carried on along this line by our research group.

Another open issue consists in the fact that the spin-polarized current $\mathbf{J}_{s}$ obtained from Equation (1) and from the experimental determination of $\mathbf{E}_{\mathrm{ISHE}}$ is found to exceed by more than one order of magnitude the value that can be derived from a standard spin drift-diffusion model [43]. This is true for the data displayed in Figure 4 but is also confirmed by the analysis of the information reported in Refs. [33] and [53]. Such an inconsistency might be due to several reasons: the application of diffusion equations for the modeling of spin relaxation in layers whose thickness is thinner or comparable with the diffusion length, the large scattering of the experimental values of $\gamma$ reported in the literature, an incorrect evaluation of the spin diffusion length $L_{s}$, or the use of the conductivity value reported for bulk materials to describe the electrical properties of the extremely thin high- $Z$ films across which $V_{\text {ISHE }}$ is measured. It is worth noticing that a similar inconsistency has been recently reported also for the ISHE obtained by spin-pumping in Pt [51] and for spin-injection through magnetic semiconductors tunnel contacts [54].

\section{Conclusions}

In conclusion, we have shown that the combination of optical orientation with ISHE is an ideal tool to address spin-dependent transport properties across homogeneous samples or metal-semiconductor Schottky junctions. Several open issues, however, still require further experimental as well as theoretical investigation in order to attain a quantitative relationship between the optically-generated spin density in the semiconductor and the ISHE effect that is measured across a high-Z metal thin film.

Acknowledgments: Funding from the CARIPLO project Grant Nos. 2011-0382 and 2013-0623 and from the SiGeSPIN project \#ANR-13-BS10-0002 is acknowledged.

Author Contributions: M.F., G.I., F.B., and F.C. conceived and designed the experiments; M.B., A.B., J.F., F.R., C.V., A.M., and M.J. fabricated the samples; F.B. and C.Z. performed the experiments; M.F., F.B., C.Z., and G.I. analyzed the data; G.I. numerically solved the diffusion model; all the authors contributed to the writing of the manuscript.

Conflicts of Interest: The authors declare no conflict of interest. 


\section{Abbreviations}

The following abbreviations are used in this manuscript:

$\mathrm{HH}$ Heavy hole

ISHE Inverse spin Hall effect

LH Light hole

MTJ Magnetic tunnel junction

SHE Spin Hall effect

SO Split-off

\section{References}

1. Žutić, I.; Fabian, J.; Das Sarma, S. Spintronics: Fundamentals and applications. Rev. Mod. Phys. 2004, 76, 323-410.

2. Tserkovnyak, Y.; Brataas, A.; Bauer, G.E.W. Enhanced Gilbert Damping in Thin Ferromagnetic Films. Phys. Rev. Lett. 2002, 88, 117601.

3. Tserkovnyak, Y.; Brataas, A.; Bauer, G.E.W.; Halperin, B.I. Nonlocal magnetization dynamics in ferromagnetic heterostructures. Rev. Mod. Phys. 2005, 77, 1375-1421.

4. Johnson, M.; Silsbee, R.H. Coupling of electronic charge and spin at a ferromagnetic-paramagnetic metal interface. Phys. Rev. B 1998, 37, 5312-5325.

5. Rashba, E.I. Theory of electrical spin injection: Tunnel contacts as a solution of the conductivity mismatch problem. Phys. Rev. B 2000, 62, R16267-R16270.

6. Fert, A.; Jaffrès, H. Conditions for efficient spin injection from a ferromagnetic metal into a semiconductor. Phys. Rev. B 2001, 64, 184420.

7. Lampel, G. Nuclear dynamic polarization by optical electronic saturation and optical pumping in semiconductors. Phys. Rev. Lett. 1968, 20, 491-493.

8. Meier, F.; Zakharchenya, B.P. Optical Orientation. Mod. Probl. Condens. Matter Sci. 1984, 8, 1-523.

9. Pierce, D.; Meier, F. Photoemission of spin-polarized electrons from GaAs. Phys. Rev. B 1976, 13, 5484-5500.

10. Allenspach, R.; Meier, F.; Pescia, D. Experimental Symmetry Analysis of Electronic States by Spin-Dependent Photoemission. Phys. Rev. Lett. 1983, 51, 2148-2150.

11. Bottegoni, F.; Isella, G.; Cecchi, S.; Ciccacci, F. Spin polarized photoemission from strained Ge epilayers. Appl. Phys. Lett. 2011, 98, 242107.

12. Bottegoni, F.; Ferrari, F.; Isella, G.; Finazzi, M.; Ciccacci, F. Enhanced orbital mixing in the valence band of strained germanium. Phys. Rev. B 2012, 85, 245312.

13. Ferrari, A.; Bottegoni, F.; Isella, G.; Cecchi, S.; Ciccacci, F. Epitaxial $\mathrm{Si}_{1-x} \mathrm{Ge}_{x}$ alloys studied by spin-polarized photoemission. Phys. Rev. B 2013, 88, 115209.

14. Dal Conte, S.; Bottegoni, F.; Pogna, E.A.A.; De Fazio, D.; Ambrogio, S.; Bargigia, I.; D’Andrea, C.; Lombardo, A.; Bruna, M.; Ciccacci, F.; et al. Ultrafast valley relaxation dynamics in monolayer $\mathrm{MoS}_{2}$ probed by nonequilibrium optical techniques. Phys. Rev. B 2015, 92, 235425.

15. Bottegoni, F.; Celebrano, M.; Bollani, M.; Biagioni, P.; Isella, G.; Ciccacci, F.; Finazzi, M. Spin voltage generation through optical excitation of complementary spin populations. Nat. Mater. 2014, 13, 790-795.

16. Kikkawa, J.M.; Awschalom, D.D. Resonant Spin Amplification in n-Type GaAs. Phys. Rev. Lett. 1998, 80, 4313-4316.

17. Pezzoli, F.; Bottegoni, F.; Trivedi, D.; Ciccacci, F.; Giorgioni, A.; Li, P.; Cecchi, S.; Grilli, E.; Song, Y.; Guzzi, M.; et al. Optical Spin Injection and Spin Lifetime in Ge Heterostructures. Phys. Rev. Lett. 2012, $108,156603$.

18. Hilton, D.J.; Tang, C.L. Optical Orientation and Femtosecond Relaxation of Spin-Polarized Holes in GaAs. Phys. Rev. Lett. 2002, 89, 146601.

19. Loren, E.J.; Rioux, J.; Lange, C.; Sipe, J.E.; van Driel, H.M.; Smirl, A.L. Hole spin relaxation and intervalley electron scattering in germanium. Phys. Rev. B 2011, 84, 214307.

20. D'yakonov, M.I.; Perel', V.I. Spin relaxation of conduction electrons in noncentrosymmetric semiconductors. Sov. Phys. Solid State 1972, 13, 3023-3026.

21. Bottegoni, F.; Drouhin, H.-J.; Fishman, G.; Wegrowe, J.-E. Probability- and spin-current operators for effective Hamiltonians Phys. Rev. B 2012, 85, 235313. 
22. Bottegoni, F.; Drouhin, H.-J.; Wegrowe, J.-E.; Fishman, G. Probability-current definition in presence of spin-orbit interaction. J. App. Phys. 2012, 111, 07 C305.

23. Allenspach, R.; Meier, F.; Pescia, D. Spin polarized photoemission from GaAs and Ge: Temperature dependence of the threshold polarization. Appl. Phys. Lett. 1984, 44, 1107-1109.

24. Parsons, R.R. Band-To-Band Optical Pumping in Solids and Polarized Photoluminescence. Phys. Rev. Lett. 1969, 23, 1152-1154.

25. Pezzoli, F.; Qing, L.; Giorgioni, A.; Isella, G.; Grilli, E.; Guzzi, M.; Dery, H. Spin and energy relaxation in germanium studied by spin-polarized direct-gap photoluminescence. Phys. Rev. B 2013, 88, 045204.

26. Jullière, M. Tunneling between ferromagnetic films. Phys. Lett. 1975, 54A, 225-226.

27. D'yakonov, M.I.; Perel', V.I. Possibility of Orienting Electron Spins with Current. JETP Lett. 1971, 13, 467-469.

28. Saitoh, E.; Ueda, M.; Miyajima, H.; Tatara, G. Conversion of spin current into charge current at room temperature: Inverse spin-Hall effect. Appl. Phys. Lett. 2006, 88, 182509.

29. Bakun, A.A.; Zakharchenya, B.P.; Rogachev, A.A.; Tkachuk, M.N.; Fleisher, V.G. Observation of a surface photocurrent caused by optical orientation of electrons in a semiconductor. JETP Lett. 1984, 40, 1293-1295.

30. Bottegoni, F.; Ferrari, A.; Isella, G.; Finazzi, M.; Ciccacci, F. Experimental evaluation of the spin-Hall conductivity in Si-doped GaAs. Phys. Rev. B 2013, 88, 121201(R).

31. Ando, K.; Morikawa, M.; Trypiniotis, T.; Fujikawa, Y.; Barnes, C.H.W.; Saitoh, E. Photoinduced inverse spin-Hall effect: Conversion of light-polarization information into electric voltage. Appl. Phys. Lett. 2010, 96, 082502 .

32. Bottegoni, F.; Ferrari, A.; Cecchi, S.; Finazzi, M.; Ciccacci, F.; Isella, G. Photoinduced inverse spin Hall effect in Pt/Ge(001) at room temperature. Appl. Phys. Lett. 2013, 102, 152411.

33. Ando, K.; Morikawa, M.; Trypiniotis, T.; Fujikawa, Y.; Barnes, C.H.W.; Saitoh, E. Direct conversion of light-polarization information into electric voltage using photoinduced inverse spin-Hall effect in Pt/GaAs hybrid structure: Spin photodetector. J. Appl. Phys. 2010, 107, 113902.

34. Kato, Y.K.; Myers, R.C.; Gossard, A.C.; Awschalom, D.D. Observation of the spin Hall effect in semiconductors. Science 2004, 5703, 1910-1913.

35. Kato, Y.K.; Myers, R.C.; Gossard, A.C.; Awschalom, D.D. Current-Induced Spin Polarization in Strained Semiconductors. Phys. Rev. Lett. 2004, 93, 5326-5335.

36. Wunderlich, J.; Kaestner, B.; Sinova, J.; Jungwirth, T. Experimental Observation of the Spin-Hall Effect in a Two-Dimensional Spin-Orbit Coupled Semiconductor System. Phys. Rev. Lett. 2005, 94, 047204.

37. Averkiev, N.S.; D'yakonov, M.I. Current due to the inhomogeneity of the spin orientation of electrons in a semiconductor. Sov. Phys.: Semicond. 1983, 17, 393.

38. Mott, N.F. The Scattering of Fast Electrons by Atomic Nuclei. Proc. R. Soc. A-Math. Phys. Eng. Sci. 1929, $794,425-442$.

39. Chazalviel, J.-N. Spin-dependent Hall effect in semiconductors. Phys. Rev. B 1975, 11, 3918-3934.

40. Engel, H.-A.; Halperin, B.I.; Rashba, E. Theory of Spin Hall Conductivity in $n$-Doped GaAs. Phys. Rev. Lett. 2005, 95, 166605.

41. Berger, L. Side-Jump Mechanism for the Hall Effect of Ferromagnets. Phys. Rev. B 1970, 11, 4559-4566.

42. Matsuzaka, S.; Ohno, Y.; Ohno, H. Electron density dependence of the spin Hall effect in GaAs probed by scanning Kerr rotation microscopy. Phys. Rev. B 2009, 80, 241305.

43. Isella, G.; Bottegoni, F.; Ferrari, A.; Finazzi, M.; Ciccacci, F. Photon energy dependence of photo-induced inverse spin-Hall effect in Pt/GaAs and Pt/Ge. Appl. Phys. Lett. 2015, 106, 232402.

44. Khamari, S.K.; Dixit, V.K.; Oak, S.M. Numerical simulation of inverse spin Hall spectra in Pt/GaAs hybrid structure. J. Phys. D: Appl. Phys. 2011, 44, 265104.

45. Hilsum, C. Simple empirical relationship between mobility and carrier concentration. Electron. Lett. 1974, 10, 259-260.

46. Aspnes, D.E.; Studna, A.A. Dielectric functions and optical parameters of Si, Ge, GaP, GaAs, GaSb, InP, InAs, and InSb from 1.5 to $6.0 \mathrm{eV}$. Phys. Rev. B 1983, 27, 985-1009.

47. Nastos, F.; Rioux, J.; Strimas-Mackey, M.; Mendoza, B.S.; Sipe, J.E. Full band structure LDA and k · p calculations of optical spin-injection. Phys. Rev. B 2007, 76, 205113.

48. Rioux, J.; Sipe, J.E. Optical injection and control in germanium: Thirty-band k · p theory. Phys. Rev. B 2010, $81,155215$. 
49. Shockley, W.; Read, W.T. Statistics of the Recombinations of Holes and Electrons. Phys. Rev. 1952, 87, 835-842.

50. Bottegoni, F.; Ferrari, A.; Rortais, F.; Vergnaud, C.; Marty, A.; Isella, G.; Finazzi, M.; Jamet, M.; Ciccacci, F. Spin diffusion in Pt as probed by optically generated spin currents. Phys. Rev. B 2015, 92, 214403.

51. Castel, V.; Vlietstra, N.; Ben Youssef, J.; van Wees, B.J. Platinum thickness dependence of the inverse spin-Hall voltage from spin pumping in a hybrid yttrium iron garnet/platinum system. Appl. Phys. Lett. 2012, 101, 132414.

52. Schmidt, G.; Ferrand, D.; Molenkamp, L.W.; Filip, A.T.; van Wees, B.J. Fundamental obstacle for electrical spin injection from a ferromagnetic metal into a diffusive semiconductor. Phys. Rev. B 2000, 62, R4790-R4793.

53. Khamari, S.K.; Porwal, S.; Dixit, V.K.; Sharma, T.K. Temperature dependence of the photo-induced inverse spin Hall effect in Au/InP hybrid structures. Appl. Phys. Lett. 2014, 104, 042102.

54. Oltscher, M.; Ciorga, M.; Utz, M.; Schuh, D.; Bougeard, D.; Weiss, D. Electrical Spin Injection into High Mobility 2D Systems. Phys. Rev. Lett. 2014, 113, 236602.

(C) 2016 by the authors; licensee MDPI, Basel, Switzerland. This article is an open access article distributed under the terms and conditions of the Creative Commons Attribution (CC-BY) license (http://creativecommons.org/licenses/by/4.0/). 